\title{
Hacia el control radical de las enfermedades respiratorias: un alto en el camino
}

\author{
Toward an integrative control of chronic respiratory diseases in Chile: \\ contribution of social determinants on health
}

Regularmente accedemos a información cientifica que advierte sobre la creciente carga de enfermedades crónicas no transmisibles en todo los países del mundo (NCD: Non comunicable diseases), particularmente en países en vías de desarrollo o que ya lo están ${ }^{1}$.

Para nosotros esto no constituye mayor novedad, habiéndose reflexionado previamente en esta Revista sobre este fenómeno, destacando particularmente la importancia que las enfermedades crónicas del sistema respiratorio tienen y tendrán, en base al análisis de su tendencia ${ }^{2,3}$.

La reacción de los países frente a este desafío de marca mayor ha sido diversa, predominando respuestas más cercanas a la perspectiva clínica por sobre respuestas con visión poblacional, traducido esto en los siguientes hechos positivos: más y mejores terapias y oportunidades de diagnóstico, innovación e incorporación de tecnología en salud, fortalecimiento del recurso humano, diversidad de protocolos, normas, guías y orientaciones que buscan el mejor desempeño clínico posible para mejorar a nuestros enfermos y mejorar su calidad de vida y extenderla.

Las Sociedades Americana del Tórax (ATS) y Europea de Enfermedades Respiratorias (ERS), por citar un ejemplo del mundo desarrollado, han divulgado lineamientos regulares en relación a las enfermedades respiratorias crónicas más relevantes por su frecuencia como son EPOC y Asma bronquial ${ }^{4-6}$. No obstante, pareciera ser que estos esfuerzos no logran conseguir el impacto esperado y el resultado de esta modalidad de respuesta, se traduce objetivamente en una contribución marginal por parte de los sistemas de atención en el logro de mejor saludi. Los documentos señalados, suelen desarrollar minimamente aspectos preventivos de fondo, si se les da una lectura dirigida.

En materia de financiamiento en salud, es también un dogma que la disponibilidad de recursos económicos para el control de las enfermedades crónicas se hará aún más crítica, pese a recurrir a complejas y eficientes metodologías actualmente disponibles para la asignación de recursos ${ }^{7}$.

Este panorama fundamentó la necesidad de reflexionar sobre nuevas y alternativas maneras de comprender este complejo problema, buscando respuestas más integrales. La Comisión de Determinantes Sociales en Salud (DSS), convocada por la OMS $S^{8}$, tuvo el mérito de conceptualizar, operativizar $y$ difundir este conceptoii, pasando así de un ejercicio académico, a una herramienta de gestión en el ámbito de políticas públicas.

La comisión visibilizó la importancia de las siguientes circunstancias a considerar en el control radical del problema de las enfermedades crónicas: gradiente social, estrés psicosocial, los primeros años de vida y la educación, desempleo, apoyo social, adicciones (alcohol, tabaco, drogas), alimentación y transporte. Finalmente, propuso un marco de acción explícito para actuar sobre ellos ${ }^{9}$.

\footnotetext{
Se estima que el impacto global de la acción de los sistemas de atención de salud, gravita no más allá de 20 a $30 \%$ en cuanto a explicar mejorías en salud.

ii Entendidos como las circunstancias en que las personas nacen, crecen, viven, trabajan y envejecen, incluido el sistema de salud.
} 
En el ámbito de la medicina respiratoria, y atingente a este informe y su análisis, hay evidencia contundente en relación a disparidad en el acceso a diagnóstico de estas enfermedades, desiguales resultados explicados por razones no médicas ${ }^{10}$, desigualdad de acceso según género, raza, edad ${ }^{11,12}$. Más dramáticamente, el acceso a medicación respiratoria básica, pareciera no ser del todo adecuada, introduciendo como factor de desigualdad, la propia acción de los servicios de atención de salud por inadecuadas prácticas de gestión ${ }^{13}$.

Es urgente un mayor entusiasmo en los profesionales abocados al control de las enfermedades respiratorias, para abogar en el control de las denominadas "causas de las causas de estas enfermedades", explorando los DSS. Ya parece no bastar con la sola indicación de cesación del hábito de fumar, siendo preciso estudiar, comprender y modificar las causas por las cuales las personas fuman, incluyendo la disposición del Estado a intervenir complementariamente, caso emblemático en el ámbito de las enfermedades respiratorias.

El enfoque de los DSS ha tenido el mérito de permitir una reflexión sobre denominaciones, definiciones y supuestos que hoy son objeto de debate intenso, entre las que podemos mencionar lo siguiente: las enfermedades crónicas pueden trasmitirse, quizás no biológicamente, pero sí, conductualmente; no son territorio exclusivo de los adultos, lo son también del niño; su comienzo puede estar in utero y su control, por tanto, comienza en el período prenatal; intervenciones desacertadas en el crecimiento y desarrollo del niño pueden afectar adversamente al futuro adulto; la descompensación aguda, es tan relevante como la cronicidad en sí misma y objeto de prioridad; el manejo clínico de la enfermedad respiratoria crónica debe ser multidisciplinario, si se desea alcanzar la plenitud de beneficios clínicos.

La Sociedad Chilena de Enfermedades Respiratoria ha dado importantes pasos para asumir el desafio de reflexionar críticamente sobre el papel que le compete en el control integral de las enfermedades respiratorias y en especial en la lucha contra las enfermedades crónicas ${ }^{14-17}$.

A la excelencia académica de sus reuniones y congresos cientificos regulares, se suma la inquietud manifiesta por abordar temáticas relevantes. Resta avanzar hacia el ponderado equilibrio entre el conocimiento científico, las prácticas clínicas de excelencia y el involucramiento en el diseño de políticas públicas que consideren los DSS para una acción más radical y de mayor impacto.

\section{Dr. Gonzalo Valdivia $C$. Profesor Titular, Depto. de Salud Pública Pontificia Universidad Católica de Chile. Miembro del Comité Científico, Sociedad Chilena de Enfermedades Respiratorias. Email: valdiviacg@gmail.com}

\section{Bibliografía}

1.- LOZANO R, NAGHAVI M, FOREMAN K, LIM S, SHIBUYA K, ABOYANS V, et al. Global and regional mortality from 235 causes of death for 20 age groups in 1990 and 2010: a systematic analysis for the Global Burden of Disease Study 2010. The Lancet 2013; 380: 2095-128.

2.- VALDIVIA G. Encuesta nacional de salud 2009-2010: Enseñanzas y desafíos. Rev Chil Enferm Respir 2011; 27: 5-6.

3.- CALVO M. Relaciones de la Sociedad Chilena de Enfermedades Respiratorias con el Ministerio de Salud. Rev Chil Enferm Respir 2014; 30: 195-6.

4.- PAUWELS R, BUIST S, CALVERLEY P, JENKINS C,
HURD S. Global Strategy for the Diagnosis, Management, and Prevention of Chronic Obstructive Pulmonary Disease. Am J Respir Crit Care Med 2001; 163: 125676.

5.- VESTBO J, HURD S, AGUSTÍ A, JONES P, VOGELMEIER C, ANZUETO A, et al. Global Strategy for the Diagnosis, Management, and Prevention of Chronic Obstructive Pulmonary Disease. Am J Respir Crit Care Med 2013; 187: 347-65.

6.- AMERICAN THORACIC SOCIETY. Documents/European Respiratory Society Statement: Asthma Control and Exacerbations Standardizing Endpoints for Clinical Asthma Trials and Clinical Practice. Am J Respir Crit Care Med 2009; 180: 59-99.

7.- BANTA D, KRISTENSEN FB, JONSSON E. A history 
of health technology assessment at the European level. Internat J Technol Assess Health Care 2009; 25 (Suppl 1): $68-73$

8.- ORGANIZACIÓN MUNDIAL DE LA SALUD. Comisión Sobre Determinantes Sociales de la Salud. Informe Final: Subsanar las Desigualdades en una Generación. 2008. Disponible en: http://www.who.int/social_determinants/thecommission/finalreport/en/. Consultado el 16 de abril de 2015.

9.- ORGANIZACIÓN MUNDIAL DE LA SALUD. Comisión sobre Determinantes Sociales de la Salud | Resumen analítico del informe final. WHO/IER/CSDH/08.1. Ginebra, 2008. Disponible en: http://whqlibdoc.who.int/ hq/2008/WHO_IER_CSDH_08.1_spa.pdf. Consultada el 15 de abril de 2015 .

10.- GERSHON A S, DOLMAGE T E, STEPHENSON A, JACKSON B. Chronic Obstructive Pulmonary Disease and SocioEconomic Status: a Systematic Review. COPD 2012; 9: 216-26.

11.- MURRAY J. Inequities in lung health: challenges and solutions. (Ed) Int J Tuber Lung Dis 2010; 14: 931-4
12.- EISNER M D, BLANC P D, OMACHI T A, YELIN E H, SIDNEY S, KATZ P P, et al Socioeconomic status, race and COPD health outcomes. J Epidemiol Community Health 2011; 65: 26-34.

13.- BLANC P, EISNER M, YELIN E, EARNEST G, BALMES JR, GREGORICH SE, et al Socioeconomic gradients in tiotropium use among adults with COPD. Int J Chron Obstruct Pulmon Dis 2008; 3: 483-90.

14.- OYARZÚN M, VALDIVIA G. Contaminación atmosférica: Declaración de la Academia de Medicina, Instituto de Chile. Rev Chil Enferm Respir 2012; 28: 171-3.

15.- SULBRANDT J, PINO P, OYARZÚN M. Envejecimiento activo y saludable: investigación y políticas para el envejecimiento poblacional. Rev Chil Enferm Respir 2012; 28: 269-71.

16.- DÍAZ P, UNDURRAGAA. Uso y abuso de los corticoides en las enfermedades respiratorias. Rev Chil Enferm Respir 2013; 29: 67-9.

17.- CONTRERAS G, TORREALBA B. Enfermedades respiratorias ocupacionales. Rev Chil Enferm Respir 2013; 29: $189-90$. 